\title{
Anomalous Self-Energy Features in the 2D Hubbard Model
}

\author{
A. Avella and F. Mancini \\ Dipartimento di Fisica "E.R. Caianiello" — Unità CNISM di Salerno \\ Università degli Studi di Salerno, 84081 Baronissi (SA), Italy \\ The electronic self-energy of the two-dimensional Hubbard model, com- \\ puted in the non-crossing approximation within the composite operator \\ method, is studied as a function of frequency as well as temperature at \\ the Fermi surface, for a value of doping at which the pseudogap is already \\ well developed. For values of momentum belonging to the phantom arc of \\ the Fermi surface, both dependences show anomalous power law behavior in \\ contrast to that expected from the Fermi liquid theory.
}

PACS numbers: 71.10.Fd, 71.27.+a, 71.10.--w

\section{Introduction}

The two-dimensional Hubbard model is generally believed to be the minimal model sufficient to describe various aspects of high- $T_{\mathrm{c}}$ cuprate superconductors - strong electronic correlations, competition between localization and itineracy, Mott physics, and low-energy spin excitations. At the same time, certain anomalous features - pseudogap, Fermi arcs, kinks, non-Fermi liquid behavior seen experimentally in the underdoped region of the phase diagram, call for use of powerful self-consistent analytical approximation methods. In this work, encouraged by rather promising results obtained in Ref. [1] by means of the non-crossing approximation within the composite operator method, we analyze the frequency and temperature dependence of the electronic self-energy at the Fermi surface in the underdoped region.

\section{The Hubbard model}

The Hamiltonian of the two-dimensional Hubbard model reads as

$$
H=-4 t \sum_{\boldsymbol{i j}} \alpha_{\boldsymbol{i j}} c^{\dagger}(\boldsymbol{i}) c(\boldsymbol{j})+U \sum_{\boldsymbol{i}} n_{\uparrow}(\boldsymbol{i}) n_{\downarrow}(\boldsymbol{i})-\mu \sum_{\boldsymbol{i}} n(\boldsymbol{i}),
$$

where $c^{\dagger}(\boldsymbol{i})=\left(c_{\uparrow}^{\dagger}(\boldsymbol{i}), c_{\downarrow}^{\dagger}(\boldsymbol{i})\right)$ is the electronic creation operator in spinorial notation at the site $\boldsymbol{i}, n_{\sigma}(\boldsymbol{i})=c_{\sigma}^{\dagger}(\boldsymbol{i}) c_{\sigma}(\boldsymbol{i})$ is the charge density operator for spin $\sigma$ at the site $\boldsymbol{i}, n(\boldsymbol{i})=\sum_{\sigma} n_{\sigma}(\boldsymbol{i})$ is the charge density operator at the site $\boldsymbol{i}, \boldsymbol{\mu}$ is the chemical potential, $U$ is the on-site Coulomb interaction strength, $t$ is the hopping integral, 
$\alpha_{i j}$ is the projection operator on the nearest-neighbor sites. (For a generic operator $\Psi(\boldsymbol{i})$, we will often use the following definition: $\Psi^{\alpha}(\boldsymbol{i})=\sum_{\boldsymbol{j}} \alpha_{\boldsymbol{i} \boldsymbol{j}} \Psi(\boldsymbol{j})$.)

\section{The equations of motion and the basic field}

We begin with the following equation of motion for the electronic operator $c(i)$ in the Heisenberg picture $(i=(\boldsymbol{i}, t))$ :

$$
\mathrm{i} \frac{\partial}{\partial t} c(i)=-\mu c(i)-4 t c^{\alpha}(i)+U \eta(i)
$$

with $\eta(i)=n(i) c(i)$ and decompose $c(i)$ as $c(i)=\xi(i)+\eta(i)$, where $\xi(i)=$ $[1-n(i)] c(i)$ and choose $\xi(i)$ and $\eta(i)$ as components of the basic field $\psi^{\dagger}(i)=$ $\left(\xi^{\dagger}(i), \eta^{\dagger}(i)\right)$.

The basic field then satisfies the following equations of motion:

$$
\mathrm{i} \frac{\partial}{\partial t} \psi(i)=\sum_{\boldsymbol{j}} \varepsilon_{\boldsymbol{i j}} \psi(\boldsymbol{j}, t)+\delta J(i)
$$

with

$$
\begin{aligned}
& \varepsilon_{\boldsymbol{i j}}=\delta_{\boldsymbol{i} \boldsymbol{j}} \varepsilon_{0}-4 t \alpha_{\boldsymbol{i} \boldsymbol{j}} I\left(1+\sigma_{1}\right), \\
& \delta J(i)=\sigma_{3} \pi(i), \quad \pi(i)=\frac{1}{2} \sigma^{\mu} \delta n_{\mu}(i) c^{\alpha}(i)+c^{\dagger \alpha}(i) c(i) c(i), \\
& \varepsilon_{0}=\left(\begin{array}{cc}
-\mu & 0 \\
0 & U-\mu
\end{array}\right), \quad I=\mathcal{F}\left\langle\left\{\psi(\boldsymbol{i}), \psi^{\dagger}(\boldsymbol{j})\right\}\right\rangle=\left(\begin{array}{cc}
1-\frac{n}{2} & 0 \\
0 & \frac{n}{2}
\end{array}\right),
\end{aligned}
$$

where $n$ is the filling, $\delta n_{\mu}(i)=c^{\dagger}(i) \sigma_{\mu} c(i)-\delta_{\mu 0} n$ is the charge $(\mu=0)$ and spin $(\mu=1,2,3)$ density fluctuation operator at the site $\boldsymbol{i}, \sigma_{\mu}=(1, \boldsymbol{\sigma}), \sigma^{\mu}=(-1, \boldsymbol{\sigma}), \boldsymbol{\sigma}$ are the Pauli matrices, $\langle\ldots\rangle$ stands for the thermal average in the grand-canonical ensemble and $\mathcal{F}$ denotes the Fourier transform.

\section{The Green function}

We now compute the thermal retarded Green function $G(\boldsymbol{k}, \omega)=$ $\mathcal{F}\left\langle\mathcal{R}\left[\psi(i) \psi^{\dagger}(j)\right]\right\rangle$ in terms of the self-energy $\Sigma(\boldsymbol{k}, \omega)$ and, in turn, of the scattering matrix $T(\boldsymbol{k}, \omega)=I^{-1}\left[m(\boldsymbol{k})+\mathcal{F}\left\langle\mathcal{R}\left[\delta J(i) \delta J^{\dagger}(j)\right]\right\rangle\right) I^{-1}$ :

$$
G(\boldsymbol{k}, \omega)=\frac{1}{\omega-\varepsilon(\boldsymbol{k})-\Sigma(\boldsymbol{k}, \omega)} I, \quad \Sigma(\boldsymbol{k}, \omega)=I \frac{1}{T^{-1}(\boldsymbol{k}, \omega)+G_{0}(\boldsymbol{k}, \omega)},
$$

where $G_{0}(\boldsymbol{k}, \omega)=\frac{1}{\omega-\varepsilon(\boldsymbol{k})} I$ is just the 0-th approximation $(\delta J(\boldsymbol{i})=0)$ and $m(\boldsymbol{k})=\mathcal{F}\left\langle\left\{\delta J(\boldsymbol{i}), \psi^{\dagger}(\boldsymbol{j})\right\}\right\rangle=-4 t[\alpha(\boldsymbol{k}) p+\Delta]\left(1-\sigma_{1}\right)$ with $\Delta=C_{11}^{\alpha}-C_{22}^{\alpha}$, $p=\frac{1}{4}\left(C_{0}^{\alpha}+3 C_{3}^{\alpha}\right)-C_{p}^{\alpha}, \quad C^{\alpha}=\left\langle\psi^{\alpha}(\boldsymbol{i}) \psi^{\dagger}(\boldsymbol{i})\right\rangle, \quad C_{\mu}^{\alpha}=\left\langle\delta n_{\mu}^{\alpha}(\boldsymbol{i}) \delta n_{\mu}(\boldsymbol{i})\right\rangle$, $C_{p}^{\alpha}=\left\langle\left[c_{\uparrow}(\boldsymbol{i}) c_{\downarrow}(\boldsymbol{i})\right]^{\alpha} c_{\downarrow}^{\dagger}(\boldsymbol{i}) c_{\uparrow}^{\dagger}(\boldsymbol{i})\right\rangle$.

In the pole approximation, we would have just $\Sigma(\boldsymbol{k}, \omega) \approx m(\boldsymbol{k}) I^{-1}$ as we would replace $\delta J(i)$ by its projection on the chosen basis $\psi(i): \delta J(k) \approx$ $m(\boldsymbol{k}) I^{-1} \psi(k)$. In the non-crossing approximation [2], we take advantage of the possibility of exactly rewriting $\delta J(i)$ in terms of bosonic fields multiplied by fermionic ones. Accordingly, we can approximate the scattering matrix $T(\boldsymbol{k}, \omega)$ 
and retain the pole contribution plus the non-crossing diagrams, that is diagrams containing the convolution of charge and spin. (Here we choose to neglect the pair term propagators compared to the electronic one [1]):

$$
\begin{aligned}
\Sigma\left(\boldsymbol{k}, \omega_{n}\right) & \approx m(\boldsymbol{k}) I^{-1}-4 t^{2}\left(1-\sigma_{1}\right) \\
& \times \mathcal{F}\left\{\mathcal{F}^{-1}\left[S\left(\boldsymbol{k}, \omega_{n}\right)\right] \mathcal{F}^{-1}\left[\alpha^{2}(\boldsymbol{k}) G_{c c}\left(\boldsymbol{k}, \omega_{n}\right)\right]\right\} I^{-1},
\end{aligned}
$$

where

$$
G_{c c}\left(\boldsymbol{k}, \omega_{n}\right)=\mathcal{F}\left\langle\mathcal{T}_{\tau}\left[c(i) c^{\dagger}(j)\right]\right\rangle
$$

and

$$
S\left(\boldsymbol{k}, \omega_{n}\right)=\mathcal{F}\left\langle\mathcal{T}_{\tau}\left[\delta n_{\mu}(i) \delta n_{\mu}(j)\right]\right\rangle
$$

Here, the parameter $p$ has been fixed through the algebraic constraint $\left\langle\xi(\boldsymbol{i}) \eta^{\dagger}(\boldsymbol{i})\right\rangle=0[3]$ and charge and spin propagators have been computed in the two-pole approximation [4]. It is worth noticing that this approach is completely microscopic, exclusively analytical, and fully self-consistent.

\section{Results}

In Fig. 1, we can see that the imaginary part of the self-energy $\Sigma^{\prime \prime}(\underline{\boldsymbol{k}}, \omega)$ shows two different behaviors as a function of frequency when computed at the nodal point $\underline{\boldsymbol{k}}=S$ and at its companion position on the phantom half of the hole pocket $\underline{\boldsymbol{k}}=\underline{S}$ (see Fig. 2 (left part) and Ref. [1]). In particular, at $\underline{\boldsymbol{k}}=S$, a parabolic-like (i.e., Fermi-liquid-like) behavior is apparent, whereas at $\underline{\boldsymbol{k}}=\underline{S}$, the dependence shows a predominance of the linear term.
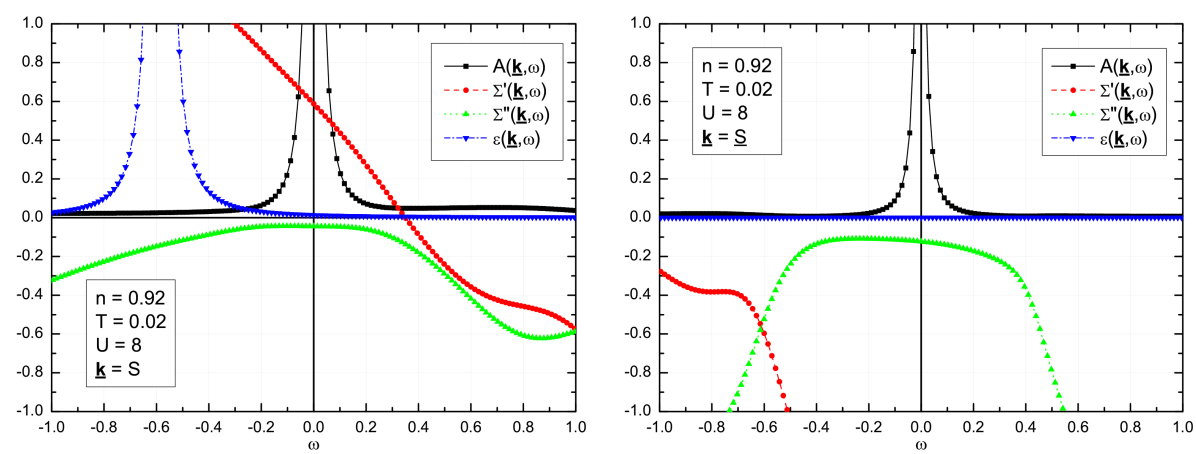

Fig. 1. Spectral density function $A(\underline{\boldsymbol{k}}, \omega)$, real (') and imaginary (") part of the self-energy $\Sigma(\underline{\boldsymbol{k}}, \omega)$, non-interacting dispersion $\varepsilon(\underline{\boldsymbol{k}}, \omega)$ as functions of frequency at (left) $\underline{\boldsymbol{k}}=S$ and (right) $\underline{\boldsymbol{k}}=\underline{S}$ for $n=0.92, T=0.02$ and $U=8$.

In Fig. 2 (right part), the imaginary part of the self-energy at the Fermi surface $\Sigma^{\prime \prime}(\underline{\boldsymbol{k}}, \omega=0)$ is reported as a function of the temperature at the nodal point $\underline{\boldsymbol{k}}=S$ and at $\underline{\boldsymbol{k}}=\underline{S}$. Although less evident than for the frequency dependence, 

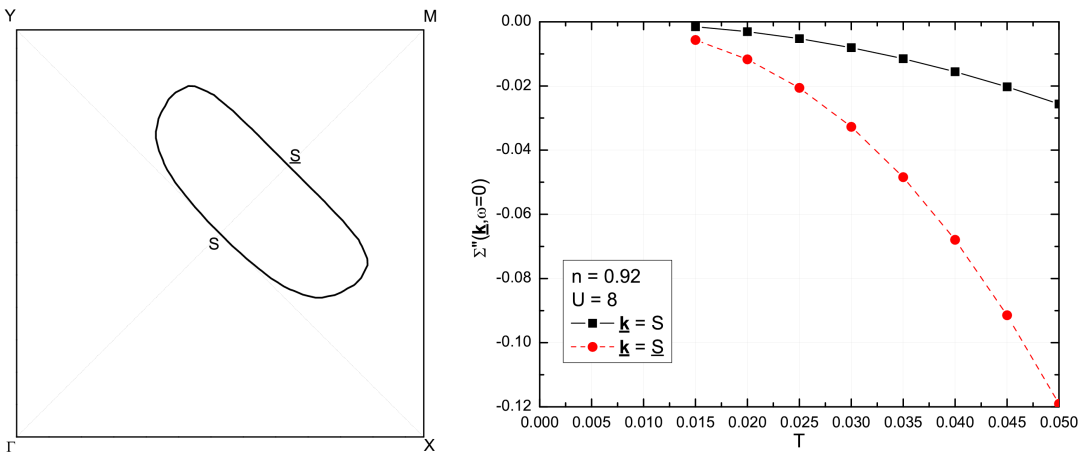

Fig. 2. (left) Underlying Fermi surface at $U=8, T=0.02$ and $n=0.92$. (right) Imaginary part of the self-energy $\Sigma^{\prime \prime}(\underline{\boldsymbol{k}}, \omega=0)$ as function of temperature at (squares) $\underline{\boldsymbol{k}}=S$ and (circles) $\underline{\boldsymbol{k}}=\underline{S}$ for $n=0.92$ and $U=8$.

the temperature dependence is also rather different: exactly parabolic (i.e., exactly Fermi-liquid) at the nodal point, with a predominance of linear and logarithmic contributions at $\underline{S}$.

\section{Conclusions}

Summarizing, we have shown that the pseudogap opening and the deconstruction of the Fermi surface in open arcs are accompanied by non-Fermi-liquid behaviors of the electronic self-energy as manifested in the frequency and temperature dependence.

\section{References}

[1] A. Avella, F. Mancini, Phys. Rev. B 75, 134518 (2007).

[2] J. Bosse, W. Götze, M. Lücke, Phys. Rev. A 17, 434 (1978).

[3] F. Mancini, A. Avella, Adv. Phys. 53, 537 (2004).

[4] A. Avella, F. Mancini, V. Turkowski, Phys. Rev. B 67, 115123 (2003). 